\title{
ASYMPTOTIC NORMALITY, STRONG MIXING AND SPECTRAL DENSITY ESTIMATES ${ }^{1}$
}

\author{
By M. RosenblatT \\ University of California, San Diego
}

\begin{abstract}
Asymptotic normality is proven for spectral density estimates assuming strong mixing and a limited number of moment conditions for the process analyzed. The result holds for a large class of processes that are not linear and does not require the existence of all moments.
\end{abstract}

1. Introduction. Strong mixing as a sufficient condition (usually together with some auxiliary moment conditions) for classical limit theorems was initially proposed and discussed in the period from the mid 50's to the early 60's (see Rosenblatt 1956, 1961; Kolmogorov and Rozanov, 1960; and Ibragimov, 1962). There had already been some investigation of the asymptotic properties of spectral density estimates. But from that time to the present day, most results on the asymptotic normality of spectral density estimates assume either a very special structure for the process (a linear process in Anderson, 1971, and Hannan, 1970) or else existence of all moments (as in Brillinger, 1975). Approximability by multilinear schemes in independent, identically distributed random variables has been considered in Rosenblatt, 1959. Here we shall show that a direct application of a central limit theorem using strong mixing together with a limited number of moment conditions will imply asymptotic normality for a large class of spectral density estimates.

2. Strong mixing and a triangular central limit theorem. Let $X=$ $\left\{X_{n}\right\}$ be a strictly stationary process. Let $\mathscr{B}_{n}=\mathscr{B}\left(X_{k}, k \leq n\right)$ be the $\sigma$-field generated by the random variables. $X_{k}, k \leq n$ and $\mathscr{F}_{m}=\mathscr{B}\left(X_{k}, k \geq m\right)$ the $\sigma$-field generated by $X_{k}, k \geq m$. The process $X=\left\{X_{n}\right\}$ is said to be strongly mixing if

$$
\sup _{B \in \mathscr{H}_{0}, F \in \mathscr{F}_{n}}|P(B F)-P(B) P(F)|=\alpha(n) \rightarrow 0
$$

as $n \rightarrow \infty$. The following central limit theorem can be established by a big blocksmall block argument just as in Rosenblatt $(1959,1961)$. This result will be assumed. It will be shown that the result can be applied to the case of spectral density estimates.

Theorem 1. Let $\left\{Y_{j}^{(n)}, j=\ldots,-1,0,1, \cdots\right\}, E Y_{j}^{(n)} \equiv 0, n=1,2, \cdots$ be a sequence of strictly stationary processes defined on the probability space of the

Received March 1983; revised December 1983.

${ }^{1}$ Research supported in part by Office of Naval Research Contract N00014-81-K-003 and National Science Foundation Grant DMS 83-12106.

AMS 1980 subject classifications. Primary 60F05; secondary 62M15.

Key words and phrases. Asymptotic normality, strong mixing, spectral density estimates, cumulants, nonlinear functions of Gaussian processes. 
strongly mixing stationary process $X=\left\{X_{n}\right\}$. Let $Y_{j}^{(n)}$ be measurable with respect to $\mathscr{F}_{j-c(n)} \cap \mathscr{B}_{j+c(n)}$ where $c(n)=o(n), c(n) \uparrow \infty$ as $n \rightarrow \infty$. Set

$$
h_{n}(b-a)=E\left|\sum_{j=a}^{b} Y_{j}^{(n)}\right|^{2} .
$$

Assume that for any two sequences $s(n), m(n)$ with $c(n)=o(m(n)), m(n) \leq n$ and $s(n) / m(n) \rightarrow 0$ one has

$$
\frac{h_{n}(m(n))}{h_{n}(s(n))} \rightarrow \infty
$$

Let $F_{n, m}(x)$ be the distribution function of

$$
\sum_{k=1}^{m} \frac{Y_{k}^{(n)}}{\left\{(n / m) h_{n}(m)\right\}^{1 / 2}}
$$

with $m=m(n)$ and $c(n)=o(m(n)), m(n)=o(n)$. Assume that

$$
\frac{n}{m(n)} \int_{|x|>\eta} x^{2} d F_{n, m(n)}(x) \rightarrow 0
$$

as $n \rightarrow \infty$ for each $\eta>0$. There are then sequences $k(n), p(n) \rightarrow \infty$ as $n \rightarrow \infty$ with $k(n) p(n) \cong n$ such that

$$
\sum_{j=1}^{n} Y_{j}(n) / \sqrt{k(n) h_{n}(p(n))}
$$

is asymptotically normally distributed with mean zero and variance one. Also if $k(n) h_{n}(p(n)) \cong h_{n}(n)$ the normalization in (2.5) can be replaced by $\sqrt{h_{n}(n)}$.

Condition (2.4) is like a Lindeberg condition. A corresponding result with this condition replaced by a stronger Liapounov condition is valid.

COROLLARY 1. The conclusion of Theorem 1 still holds if all assumptions are the same except for condition (2.4) and condition (2.4) is replaced by the following condition (2.4)'. Let

$$
\left\{h_{n}(m)\right\}^{-(2+\delta) / 2} E\left|\sum_{k=1}^{m} Y_{k}^{(n)}\right|^{2+\delta}=O(1)
$$

for $m=m(n)$ and some $\delta>0$.

Because conditions in terms of cumulants are used a brief discussion relating to them is given. Let $Z=\left(Z_{j}, j=1, \cdots, k\right)$ be a random $k$-vector with $t=$ $\left(t_{j}, j=1, \cdots, k\right)$ a $k$-vector of coefficients. The characteristic function of the random vector $Z$ is

$$
\varphi(t)=E \exp (i t \cdot Z) .
$$

Let $m=\left(m_{1}, \cdots, m_{k}\right),|m|=\sum m_{i}$. If moments up to order $s$ exist there is a Taylor expansion

$$
\varphi(t)=\sum_{|m| \leq s} \frac{i^{|m|} \mu^{(m)}}{m !} t^{m}+o\left(|t|^{s}\right)
$$


with

$$
t^{m}=\prod_{j=1}^{k} t_{j}^{m_{j}}, m !=\prod_{j=1}^{k} m_{j} !
$$

and the moments

$$
\mu^{(m)}=\mu^{\left(m_{1}, \cdots, m_{k}\right)}=E\left(\prod_{j=1}^{k} Z_{j}^{m_{j}}\right) .
$$

There is a corresponding Taylor expansion for $\log \varphi(t)$

$$
\log \varphi(t)=\sum_{|m| \leq s} \frac{i^{|m|} c^{(m)}}{m !} t^{m}+o\left(|t|^{s}\right)
$$

with the coefficients $c^{(m)}$ the cumulants of $Z$

$$
c^{(m)}=c^{\left(m_{1}, \cdots, m_{k}\right)}=\operatorname{cum}\left(Z_{1}^{m_{1}}, \cdots, Z_{k}^{m_{k}}\right) .
$$

There are identities linking the moments $\mu^{(m)},|m| \leq s$, up to order $s$ with the cumulants $c^{(m)},|m| \leq s$, up to order $s$. In fact, the moments up to order $s$ exist if and only if the cumulants up to order $s$ exist.

At times one will deal with random variables $W_{1}, \cdots, W_{J}$ given in terms of doubly indexed random variables

$$
U_{k, \ell}, \ell=1, \cdots, \ell_{k}, \quad k=1, \cdots, J
$$

by

$$
W_{k}=\prod_{\ell=1}^{k} U_{k, \ell}
$$

The object is to carry out the computation of the cumulant

$$
\operatorname{cum}\left(W_{1}, \cdots, W_{J}\right)
$$

in terms of the cumulants of the $U_{k, \text { e. }}$ Such a computation can be carried out in the following manner. Lay out the following array of the $U_{k, e}$ 's in $J$ rows

$$
\begin{array}{cccc}
U_{1,1} & U_{1,2} & \cdots & U_{1, \ell_{1}} \\
U_{2,1} & U_{2,2} & \cdots & U_{2, \ell_{2}} \\
\vdots & \vdots & & \vdots \\
U_{J, 1} & U_{J, 2} & \cdots & U_{J, \ell_{J}} .
\end{array}
$$

A partition of this array into sets $\nu_{1}, \cdots, \nu_{r}$ is called decomposable if the union of the elements in a nonvacuous proper subcollection of sets of the partition yields exactly the elements in a nonvacuous proper subset of the rows of the table. A partition is indecomposable if it is not decomposable. One can show that

$$
\operatorname{cum}\left(W_{1}, \cdots, W_{J}\right)=\sum \prod_{s=1}^{r} \operatorname{cum}\left(U_{j, \ell},(j, \ell) \in \nu_{s}\right)
$$

where the sum on the right is extended over all indecomposable partitions of the table given above (see Leonov and Shiryaev, 1959).

Let us note that if $k$ th order moments of the process $X=\left\{X_{n}\right\}$ are finite, that the cumulants

$$
\operatorname{cum}\left(X_{j}, X_{j+s_{1}}, \cdots, X_{j+s_{k-1}}\right)=r_{s_{1}, \cdots, s_{k-1}}^{(k)}
$$


are well-defined. Summability conditions on cumulants such as

$$
\sum_{s_{1}, \cdots, s_{k-1}}\left|r_{s_{1}, \ldots, s_{k-1}}^{(k)}\right|<\infty
$$

are convenient to make at times. In Brillinger (1975) and Brillinger and Rosenblatt (1967) assumptions of the form

$$
\sum_{s_{1}, \cdots, s_{k-1}}\left(1+\left|s_{j}\right|\right)\left|r_{s_{1}, \ldots, s_{k-1}}^{(k)}\right|<\infty,
$$

$j=1, \cdots, k-1$, are made for all $k \geq 2$. We shall only have to assume a limited number of the conditions (2.6) together with strong mixing.

Most central limit theorems for dependent processes making use of mixing or mixingale conditions make an assumption on the rate of decay of the mixing coefficient (see Hall and Heyde, 1980). Notice that this is not the case for the limit theorems in this paper. The conditions are basically summability conditions on cumulants. In fact, we shall later give examples of processes that satisfy the assumptions of the theorems but still have a slow rate of decay of the mixing coefficient.

3. Spectral density estimates. The assumption (2.6) for $k=2$ is just summability of the covariances

$$
\sum_{k}\left|r_{k}\right|<\infty, r_{k}=\operatorname{cov}\left(x_{j}, x_{j+k}\right) .
$$

The spectral density $f(\lambda)$ of the process is then continuous with

$$
f(\lambda)=1 / 2 \pi \sum_{k} r_{k} e^{-i k \lambda} \text {. }
$$

Covariance estimates $r_{k}^{(n)}$ in terms of a sequence of observations, $X_{1}, \ldots, X_{n}$, $E X_{k} \equiv 0$, on $X$ are given by

$$
r_{k}^{(n)}=1 / n \sum_{j=1}^{n-k} X_{j} X_{j+k}, \quad 0 \leq k \leq n,
$$

with $r_{-k}^{(n)}=r_{k}^{(n)}$. A large class of spectral density estimates $f_{n}(\lambda)$ are of the form

$$
f_{n}(\lambda)=1 / 2 \pi \sum_{k=-n+1}^{n-1} r_{k}^{(n)} w_{k}^{(n)} \cos k \lambda
$$

with

$$
w_{k}^{(n)}=a\left(k b_{n}\right), \quad a(0)=1,
$$

with $a(x)$ continuous at zero, bounded and satisfying

$$
a(x)=a(-x) .
$$

Here $b_{n} \rightarrow 0$ with $n^{-1}=o\left(b_{n}\right)$ as $n \rightarrow \infty$. The following proposition amounts to a simple remark on the asymptotic unbiasedness of a class of estimates of the form specified above.

Proposition. Let $X=\left(X_{k}\right)$ be a weakly stationary process with $E X_{k} \equiv 0$, $\sum\left|r_{k}\right|<\infty$. Assume that

$$
f_{n}(\lambda)=1 / 2 \pi \sum_{k=-n+1}^{n-1} r_{k}^{(n)} a\left(k b_{n}\right) \cos k \lambda .
$$

Let $a(x)$ be bounded, continuous at zero with $a(0)=1$ and symmetric about zero. 
Then

$$
E f_{n}(\lambda)-f(\lambda) \rightarrow 0
$$

as $b_{n} \rightarrow 0, n \rightarrow \infty$.

Notice that

$$
\begin{aligned}
E f_{n}(\lambda)-f(\lambda)= & \frac{1}{2 \pi} \sum_{|k| \leq n} r_{k}\left(\frac{n-|k|}{n}\right)\left[a\left(k b_{n}\right)-1\right] \cos k \lambda \\
& -\frac{1}{2 \pi} \sum_{|k| \leq n} \frac{|k|}{n} r_{k} \cos k \lambda-\frac{1}{2 \pi} \sum_{|k|>n} r_{k} \cos k \lambda .
\end{aligned}
$$

The absolute summability of $r_{k}$ implies that the second and third sums on the right of (3.1) tend to zero as $n \rightarrow \infty$. The assumptions on $a(\cdot)$ together with the summability of $r_{k}$ imply that the first sum on the right of (3.1) tends to zero as $b_{n} \rightarrow \infty$ and $n \rightarrow \infty$.

The following lemma will be useful in deriving some results on the asymptotic distribution of spectral density estimates.

LEMma. Let

$$
g(u)=1 / 2 \pi \sum g_{k} e^{-i k u}, \quad \sum\left|g_{k}\right|<\infty .
$$

Further assume that $a(\cdot)$ is piecewise continuous, continuous at 0 with $a(0)=1$, symmetric and such that $a(x)=O\left(|x|^{-1 / 2-c}\right)$ for some $\varepsilon>0$ as $|x| \rightarrow \infty$. Then if

$$
W_{n}(u)=1 / 2 \pi \sum w_{k}^{(n)} e^{-i k u}
$$

with $w_{k}^{(n)}=a\left(k b_{n}\right)$ it follows that

$$
b_{n} \int_{-\pi}^{\pi} W_{n}^{2}(u+\lambda) g(u) d u \rightarrow g(\lambda) \int W^{2}(u) d u
$$

as $b_{n} \rightarrow 0$ where

$$
W(u)=\frac{1}{2 \pi} \int a(u) e^{-i u \alpha} d u
$$

while

$$
\int_{-\pi}^{\pi} W_{n}(u+\lambda) W_{n}(u+\mu) g(u) d u=o\left(b_{n}^{-1}\right)
$$

as $b_{n} \rightarrow 0$ if $\lambda \neq \mu,|\lambda-\mu|<2 \pi$.

Notice that

$$
\begin{aligned}
b_{n} \int_{-\pi}^{\pi} W_{n}(u+\lambda) W_{n}(u+\mu) g(u) d u \\
=\frac{1}{2 \pi} \sum_{\ell} g_{\ell} e^{-i \ell u} \frac{1}{2 \pi} \sum_{k} b_{n} a\left(k b_{n}\right) a\left((k-\ell) b_{n}\right) e^{i k(\mu-\lambda)} .
\end{aligned}
$$


Clearly

$$
\left|\sum_{k} b_{n} a\left(k b_{n}\right) a\left((k-\ell) b_{n}\right)\right| \leq \sum b_{n}\left|a\left(k b_{n}\right)\right|^{2}
$$

and the right-hand side of (3.4) tends to

as $b_{n} \rightarrow 0$. Also, if $\mu=\lambda$

$$
\int|a(u)|^{2} d u
$$

$$
\sum_{k} b_{n} a\left(k b_{n}\right) a\left((k-\ell) b_{n}\right) \rightarrow \int|a(u)|^{2} d u
$$

as $b_{n} \rightarrow 0$ for each fixed $\ell$. This yields the limiting relationship (3.2). Let us now consider the case in which $\lambda \neq \mu,|\lambda-\mu|<2 \pi$. Relation (3.3) will follow if we can show that

$$
\sum_{k} b_{n} a\left(k b_{n}\right) a\left((k-\ell) b_{n}\right) e^{i k(\mu-\lambda)} \rightarrow 0
$$

as $b_{n} \rightarrow 0$ for each fixed $\ell$. Given $\varepsilon>0$ there is an $L(\varepsilon)$ such that

$$
\sum_{b_{n}|k|>L(\varepsilon)} b_{n}\left|a\left(k b_{n}\right)\right|^{2}<\varepsilon .
$$

The function $a(x)$ can be uniformly approximated arbitrarily well by step functions over the interval $|x| \leq L(\varepsilon)$. It is therefore enough to show that (3.5) holds for a step function $a(x)$ of finite support. In fact, this will follow if one can prove (3.5) for $a(x)$ the indicator function of a finite interval, say the interval $\left(\alpha_{1}, \alpha_{2}\right)$, $\alpha_{1}<\alpha_{2}$. Then

$$
\begin{aligned}
\sum_{k} a\left(k b_{n}\right) a\left((k-\ell) b_{n}\right) e^{i k(\mu-\lambda)} & =\sum_{\alpha_{1}<k b_{n}<\alpha_{2}} e^{i k(\mu-\lambda)} b_{n}+O\left(b_{n}\right) \\
& =e^{i \alpha_{1} c_{n}(\mu-\lambda)} \sum_{0<k b_{n}<\alpha_{2}-\alpha_{1}} e^{i k(\mu-\lambda)} b_{n}+O\left(b_{n}\right) \\
& =b_{n} e^{i \alpha_{1} c_{n}(\mu-\lambda)} \frac{e^{i\left(\alpha_{2}-\alpha_{1}\right) c_{n}(\mu-\lambda)}-1}{e^{i(\mu-\lambda)}-1}+O\left(b_{n}\right)
\end{aligned}
$$

with $c_{n}=b_{n}^{-1}$.

Let

$$
\eta(\lambda)= \begin{cases}1 & \text { if } \lambda=k \pi, \quad k \text { integer } \\ 0 & \text { otherwise. }\end{cases}
$$

We shall prove the following theorem.

THEOREM 2. Let $X=\left\{X_{n}\right\}$ be a strictly stationary strongly mixing process with $E X_{j} \equiv 0$. Assume that the cumulant functions (2.6) of order two and four are summable. Further, let the spectral density estimate $f_{n}(\lambda)$ have weights $w_{k}^{(n)}$ defined in terms of a function $a(\cdot)$ that is piecewise continuous, continuous at zero with $a(0)=1$, symmetric about zero and is such that $x a(x)$ is bounded. Let

$$
Y_{u}^{(n)}(\lambda)=\sum_{k=-c(n)}^{c(n)} X_{u} X_{u+k} w_{k}^{(n)} \cos k \lambda
$$


with $w_{k}^{(n)}=a(k b(n))$ and $c(n)=\alpha b_{n}^{-1}$ for all sufficiently large fixed $\alpha$. Set

$$
Z_{n}(\lambda)=\sum_{u=1}^{m} \frac{Y_{u}^{(n)}(\lambda)}{\left\{n b_{n}^{-1}\right\}^{1 / 2}}
$$

with $m=m(n)$ and $c(n)=o(m(n)), m(n)=o(n)$. Consider the distribution function $F_{n, m}(x)$ of $Z_{n}(\lambda)$ and assume that

$$
\frac{n}{m(n)} \int_{|x|>\eta} x^{2} d F_{n, m(n)}(x) \rightarrow 0
$$

as $n \rightarrow \infty, b_{n} \rightarrow 0, n b_{n} \rightarrow \infty$ for each $\eta>0$. Then $f_{n}(\lambda)-E f_{n}(\lambda)$ is asymptotically normally distributed with mean zero and variance

$$
\frac{2 \pi(1+\eta(\lambda))}{n b_{n}} f^{2}(\lambda) \int W^{2}(\alpha) d \alpha .
$$

Let us first consider the case in which $a(\cdot)$ has finite support. We first consider replacing $f_{n}(\lambda)$ by

$$
\tilde{f}_{n}(\lambda)=\frac{1}{2 \pi} \sum_{k=-c(n)}^{c(n)} \frac{1}{n} \sum_{j=1}^{n} x_{j} x_{j+k} w_{k}^{(n)} \cos k \lambda .
$$

Here the support of the function $a(\cdot)$ has been taken as the interval $[-1,1]$ to simplify notation. The argument goes through in exactly the same way for any other interval. Notice that

$$
2 \pi n \tilde{f}_{n}(\lambda)=\sum_{u=1}^{n} Y_{u}^{(n)}
$$

where

$$
Y_{u}^{(n)}=\sum_{k=-c(n)}^{c(n)} X_{u} X_{u+k} w_{k}^{(n)} \cos k \lambda .
$$

Thus, the expression (3.7) is of the type discussed in Theorem 1. Now,

$$
\sigma^{2}\left(f_{n}(\lambda)-\tilde{f}_{n}(\lambda)\right) \leq \frac{1}{\pi^{2} n^{2}} \sigma^{2}\left[\sum_{k=1}^{c(n)} \sum_{j=n-k}^{n} X_{j} X_{j+k} w_{k}^{(n)} \cos k \lambda\right]
$$

and the right-hand side of (3.8) is less than

$$
\begin{gathered}
\frac{1}{\pi^{2} n^{2}} \sum_{k, k^{\prime}=1}^{c(n)} \sum_{j=n-k}^{n} \sum_{j^{\prime}=n-k^{\prime}}^{n} \\
\cdot\left\{\left|r_{j-j^{\prime}} r_{j-j^{\prime}+k-k^{\prime}}\right|+\left|r_{j-j^{\prime}-k^{\prime}} r_{j-j^{\prime}+k}\right|+\left|r_{k, j^{\prime}-j j^{\prime}+k^{\prime}-j}^{4}\right|\right\}\left|w_{k}^{(n)} w_{k^{\prime}}^{(n)}\right| \\
\leq \frac{1}{\pi^{2} n^{2}} \sum_{k, k^{\prime}=1}^{c(n)} \sum_{s} \min \left(k^{\prime}, k\right)\left\{\left|r_{s}\right|\left|r_{s+k^{\prime}-k}\right|+\left|r_{s-k^{\prime}} r_{s+k}\right|+\left|r_{k, s, s+k^{\prime}}^{(4)}\right|\right\} \\
\cdot\left|w_{k}^{(n)}\right|\left|w_{k^{\prime}}^{(n)}\right| .
\end{gathered}
$$

The assumptions on the weights $w_{k}^{(n)}$ and the function $a(\cdot)$ imply that the weights are bounded and

$$
|k|\left|w_{k}^{(n)}\right| \leq L b_{n}^{-1}
$$

for all $k$ and some constant $L$. This together with the summability of (2.6) for 
$k=2,4$ imply that

$$
\begin{aligned}
\sigma^{2}\left(f_{n}(\lambda)-\tilde{f}_{n}(\lambda)\right) & =O\left(\left(n b_{n}\right)^{-2}\right) \\
& =o\left(\left(n b_{n}\right)^{-1}\right)
\end{aligned}
$$

As we shall see this is of smaller order of magnitude than $\sigma^{2}\left(\tilde{f}_{n}(\lambda)\right)$. Let us now consider

$\sigma^{2}\left(\sum_{u=1}^{m} Y_{u}^{(n)}\right)=h_{n}(m(n))$

$$
\begin{array}{r}
=\sum_{u, u^{\prime}=1}^{m} \sum_{k, k^{\prime}=-c(n)}^{c(n)}\left\{r_{u-u^{\prime}} r_{u+k-u^{\prime}-k^{\prime}}+r_{u^{\prime}-u+k^{\prime}} r_{u^{\prime}-u-k}+r_{k, u^{\prime}-u, u^{\prime}-u+k^{\prime}}^{(4)}\right\} \\
\cdot \cos k \lambda \cos k^{\prime} \lambda w_{k}^{(n)} w_{k^{\prime}}^{(n)}
\end{array}
$$

where $m=m(n) \leq n$ with $b_{n}^{-1}=o(m(n))$ as $n \rightarrow \infty$. The first term on the right of (3.9) is

$$
(1)=\iint_{-\pi}^{\pi} \frac{\sin ^{2}(m / 2)(\alpha-\beta)}{\sin ^{2}(1 / 2)(\alpha-\beta)} f(\alpha) f(\beta)\left|\sum_{v=-c(n)}^{c(n)} w_{v}^{(n)} \cos v \lambda e^{i v \beta}\right|^{2} d \alpha d \beta .
$$

The sequence of weight functions $W_{n}(u)$ have the property

$$
\max _{|u| \leq A m^{-1}}\left|\frac{\int_{-\pi}^{\pi} W_{n}(v) W_{n}(v+u) d v}{\int_{-\pi}^{\pi} W_{n}^{2}(u) d u}-1\right| \rightarrow 0
$$

as $m=m(n) \rightarrow \infty$ for each $A>0$ since $c(n)=b_{n}^{-1}=o(m(n))$. An argument like that given on page 176 of Rosenblatt (1974) implies that

$$
(1)=2 \pi m(1+o(1)) \int_{-\pi}^{\pi} f^{2}(\beta)\left|\frac{1}{2} W_{n}(\beta+\lambda)+\frac{1}{2} W_{n}(\beta-\lambda)\right|^{2} d \beta
$$

holds. The lemma then yields

$$
(1)=\pi m(1+\eta(\lambda)+o(1)) f^{2}(\lambda) \int_{-\pi}^{\pi} W_{n}^{2}(v) d v .
$$

The second term on the right of (3.9) is

$$
\begin{aligned}
(2)= & \iint_{-\pi}^{\pi} \frac{\sin ^{2}(m / 2)(\alpha-\beta)}{\sin ^{2} 1 / 2(\alpha-\beta)} f(\alpha) f(\beta)\left(\frac{1}{2} W_{n}(\alpha+\lambda)+\frac{1}{2} W_{n}(\alpha-\lambda)\right) \\
& \cdot\left(\frac{1}{2} W_{n}(\beta+\lambda)+\frac{1}{2} W_{n}(\beta-\lambda)\right) d \alpha d \beta .
\end{aligned}
$$

Property (3.10) of the family of weight functions as well as the continuity of $f$ imply that relation (3.11) holds for (2) as well as (1). This implies that we get estimate (3.12) valid for (2) as well as (1). A direct estimate shows that

$$
|(3)| \leq m \sum_{k, s, k^{\prime}}\left|r_{k, s, k^{\prime}}^{(4)}\right|
$$


and this is of smaller order than (3.12) under the assumptions made. Notice that

$$
\int_{-\pi}^{\pi} W_{n}(u)^{2} d u=(1+o(1)) b_{n}^{-1} \int W^{2}(u) d u .
$$

The estimates of (1), (2) and (3) imply that $\sigma^{2}\left(\tilde{f}_{n}(\lambda)\right)$ and therefore $\sigma^{2}\left(f_{n}(\lambda)\right)$ have the same asymptotic behavior as (3.1) as $n \rightarrow \infty$. This is obtained by setting $m(n)=n$. Also notice that if $m(n)=o(n)$ and $k(n) m(n)=n$, that then

$$
k(n) h_{n}(m(n)) \cong h_{n}(n)
$$

because of the summability of the cumulant functions for $k=2$ and $k=4$.

Assumption (3.6) and Theorem 1 now imply the conclusion of this theorem in the case of a function $a(\cdot)$ with finite support.

We now indicate how the argument can be modified so as to take care of a function $a(\cdot)$ that is not of finite support. One can replace $f_{n}(\lambda)$ by

$$
\hat{f}_{n}(\lambda)=1 / 2 \pi \sum_{|k| \leq c(n)} r_{k}^{(n)} w_{k}^{(n)} \cos k \lambda
$$

with error term

$$
g_{n}(\lambda)=1 / 2 \pi \sum_{c(n)<|k| \leq n} r_{k}^{(n)} w_{k}^{(n)} \cos k \lambda
$$

and

$$
c(n)=L b_{n}^{-1}
$$

where $L$ is fixed but sufficiently large. The asymptotic normality of $\hat{f}_{n}(\lambda)$ has already been determined by the argument given since it corresponds to a function $a(\cdot)$ with finite support. We just have to estimate the variance of the error $g_{n}(\lambda)$ and show that it is sufficiently small if $L$ is large. That will establish the asymptotic normality of $f_{n}(\lambda)$ for $a(\cdot)$ not of bounded support. Now

$$
\begin{aligned}
\sigma^{2}\left(g_{n}(\lambda)\right)=\frac{1}{4 \pi^{2} n^{2}} \sum_{|k|,\left|k^{\prime}\right| \geq c(n)} \sum_{u, u^{\prime}=1}^{n}\left\{r_{u-u^{\prime}} r_{u-u^{\prime}+k-k^{\prime}}+r_{u-u^{\prime}-k^{\prime}} r_{u-u^{\prime}+k}\right. \\
\left.+r_{k, u^{\prime}-u, u^{\prime}-u+k^{\prime}}^{(4)}\right\} w_{k}^{(n)} w_{k^{\prime}}^{(n)} \cos k \lambda \cos k^{\prime} \lambda
\end{aligned}
$$

and this is bounded by

$$
\begin{aligned}
\frac{1}{4 \pi^{2} n} \sum_{|k|,\left|k^{\prime}\right| \geq c(n)} \sum_{s} & \left\{\left|r_{s}\right|\left|r_{s+k-k^{\prime}}\right|+\left|r_{s-k^{\prime}} r_{s+k}\right|\right. \\
& \left.+\left|r_{k, s, s+k^{\prime}}^{(4)}\right|\right\}\left|w_{k}^{(n)}\right|\left|w_{k^{\prime}}^{(n)}\right| .
\end{aligned}
$$

Notice that the terms $\left|w_{k}^{(n)}\right|=\left|a\left(k b_{n}\right)\right|$ are bounded by an absolute constant. The sum in (3.13) involving fourth cumulants is $O(1 / n)$. The other terms in (3.13) are less than or equal to

$$
\frac{1}{4 \pi^{2} n} \sum_{|k|,\left|k^{\prime}\right| \geq c(n)} 2\left|r_{k-k^{\prime}}\right|^{\left(2^{*}\right)}\left|w_{k}^{(n)}\right|\left|w_{k^{\prime}}^{(n)}\right|
$$

where $\left|r_{k}\right|^{\left(2^{*}\right)}$ represents the convolution of the sequence $\left|r_{k}\right|$ with itself. Now

$$
\left|k w_{k}^{(n)}\right|=\left|k a\left(k b_{n}\right)\right| \leq M b_{n}^{-1}
$$


with $M$ an absolute constant. This implies that the sum (3.14) is bounded by

$$
\frac{1}{n} \sum_{s}\left|r_{s}\right|^{\left(2^{*}\right)} M^{2} b_{n}^{-2} \sum_{|k| \geq c(n)}\{|k|(|k|+|s|)\}^{-1} \leq \frac{M^{2}}{n} L^{-1} b_{n} b_{n}^{-2} \sum_{s}\left|r_{s}\right|^{\left(2^{*}\right)}
$$

because of (3.15). The variance $\sigma^{2}\left(g_{n}(\lambda)\right)$ is bounded by an absolute constant multiplied by $L^{-1}\left(b_{n} n\right)^{-1}$ and this is small compared to $\left(b_{n} n\right)^{-1}$ if $L$ is large.

Joint asymptotic normality and asymptotic independence of spectral density estimates at distinct frequencies $\lambda, 0 \leq \lambda \leq \pi$, is dealt with in the following corollary.

COROLlaRY 2. Let all the assumptions of Theorem 2 be satisfied with the exception of (3.6). This is modified as follows. Consider

$$
\sum_{j=1}^{s} \alpha_{j} Z_{n}\left(\lambda_{j}\right)
$$

with the $\lambda_{j}$ distinct frequencies in $[0, \pi]$. Let $F_{n, m(n)}(x)$ be the distribution function of (3.16). Let

$$
\frac{n}{m(n)} \int_{|x|>\eta} x^{2} d F_{n, m(n)}(x) \rightarrow 0
$$

as $n \rightarrow \infty, b_{n} \rightarrow 0, n b_{n} \rightarrow \infty$ for all values of the $\alpha_{j}^{\prime}$ 's and each $\eta>0$. It then follows that $\left(n b_{n}\right)^{1 / 2}\left\{f_{n}\left(\lambda_{j}\right)-E f_{n}\left(\lambda_{j}\right)\right\}$ are jointly asymptotically independent and normally distributed with variances

$$
2 \pi\left(1+\eta\left(\lambda_{j}\right)\right) f^{2}\left(\lambda_{j}\right) \int W^{2}(\alpha) d \alpha, j=1, \cdots, s .
$$

The conditions of Corollary 2 imply that every linear combination

$$
\left(n b_{n}\right)^{1 / 2} \sum_{j=1}^{s} \alpha_{j}\left\{f_{n}\left(\lambda_{j}\right)-E f_{n}\left(\lambda_{j}\right)\right\}
$$

is asymptotically normal. That implies that the spectral estimates $\left(n b_{n}\right)^{1 / 2}\left\{f_{n}\left(\lambda_{j}\right)\right.$ - $\left.E f_{n}\left(\lambda_{j}\right)\right\}, j=1, \cdots, s$, are jointly asymptotically normal. The asymptotic orthogonality and hence asymptotic independence follows from (3.3) of the lemma.

The conditions of the form (3.17) are sometimes difficult to verify directly. The following corollary replaces these by stronger cumulant conditions that are sometimes easier to implement and interpret.

COROLlaRY 3. Let the assumptions of the corollary be satisfied except for (3.17). These are replaced by summability of the cumulant functions up to order eight. The conclusion of the Corollary then holds.

It is enough to show this in the case of a single spectral estimate. The argument for several estimates is the same except for the additional notation.

In order to apply Corollary 2 in this situation we shall verify condition (3.17) by showing that

$$
\sigma^{-4}\left(\sum_{u=1}^{m} Y_{u}^{(n)}\right) E\left|\sum_{u=1}^{m}\left(Y_{u}^{(n)}-E Y_{u}^{(n)}\right)\right|^{2}=O(1)
$$


where $Y_{u}^{(n)}=Y_{u}^{(n)}(\lambda)$, for $m=m(n)$ as $n \rightarrow \infty$. Now

$$
E\left|\sum_{u=1}^{m}\left(Y_{u}^{(n)}-E Y_{u}^{(n)}\right)\right|^{4}=\sigma^{4}\left(\sum_{u=1}^{m} Y_{u}^{(n)}\right)+\operatorname{cum}_{4}\left(\sum_{u=1}^{m} Y_{u}^{(n)}\right) .
$$

Because of the multilinear character of the cumulant

and

$$
\operatorname{cum}_{4}\left(\sum_{u=1}^{m} Y_{u}^{(n)}\right)=\sum_{u_{1}, u_{2}, u_{3}, u_{4}=1}^{m} \operatorname{cum}_{4}\left(Y_{u_{1}}^{(n)}, Y_{u_{2}}^{(n)}, Y_{u_{3}}^{(n)}, Y_{u_{4}}^{(n)}\right)
$$

$$
\begin{aligned}
\operatorname{cum}_{4}\left(Y_{u_{1}}^{(n)}, Y_{u_{2}}^{(n)}, Y_{u_{3}}^{(n)}, Y_{u_{4}}^{(n)}\right) \\
\quad=\left(\prod_{i=1}^{4} w_{k_{i}} \cos k_{i} \lambda\right) \sum_{\nu} \operatorname{cum}\left(X_{s}, s \in \nu_{1}\right) \cdots \operatorname{cum}\left(X_{s}, s \in \nu_{p}\right)
\end{aligned}
$$

where the summation over $\nu=\nu_{1} \cup \ldots \cup \nu_{p}$ is over all indecomposable partitions (see the discussion in Section 2) of the following table

$$
\begin{aligned}
& x_{u_{1}} x_{u_{1}+k_{1}} \\
& x_{u_{2}} x_{u_{2}+k_{2}} \\
& x_{u_{3}} x_{u_{3}+k_{3}} \\
& x_{u_{4}} x_{u_{4}+k_{4}} .
\end{aligned}
$$

One of the several indecomposable partitions consisting entirely of pairs leads to the sum

$$
\begin{array}{r}
\sum_{k_{i}} \sum_{u_{i}} r_{u_{1}-u_{2}} r_{u_{3}-u_{4}} r_{u_{3}-u_{1}+k_{3}-k_{1}} r_{u_{4}-u_{2}+k_{4}-k_{2}} \\
w_{k_{1}}^{(n)} \cos k_{1} \lambda w_{k_{2}}^{(n)} \cos k_{2} \lambda w_{k_{3}}^{(n)} \cos k_{3} \lambda w_{k_{4}}^{(n)} \cos k_{4} \lambda .
\end{array}
$$

Introduce $a=u_{1}-u_{2}, b=u_{3}-u_{4}, \alpha=k_{3}-k_{1}, \beta=k_{4}-k_{2}$. Expression (3.18) is bounded by

$$
\sum_{a, b, u_{1}, u_{3}, k_{1}, k_{2}, \alpha, \beta}\left|r_{a}\right|\left|r_{b}\right|\left|r_{u_{3}-u_{1}+\alpha}\right|\left|r_{u_{3}-u_{1}-b+\alpha+\beta}\right|\left|w_{k_{1}}^{(n)}\right|\left|w_{k_{1}+\alpha}^{(n)}\right|\left|w_{k_{2}}^{(n)}\right|\left|w_{k_{2}+\beta}^{(n)}\right| \text {. }
$$

If one first sums over $k_{1}, k_{2}$ the bound

$$
b_{n}^{-2} \sum_{a, b, u_{1}, u_{3}, \alpha, \beta}\left|r_{a}\right|\left|r_{b}\right|\left|r_{u_{3}-u_{1}+\alpha}\right|\left|r_{u_{3}-u_{1}-b+\alpha+\beta}\right|
$$

is obtained. A sum over $\alpha, \beta$ yields the bound

$$
b_{n}^{-2} \sum_{a, b, u_{1}, u_{3}}\left|r_{a}\right|\left|r_{b}\right| \text {. }
$$

The sum over $a, b$ and then $u_{1}, u_{3}$ gives us the final bound

$$
b_{n}^{-2} m^{2} \text {. }
$$

All the other indecomposable partitions consisting entirely of pairs lead to the same bound. Indecomposable partitions which do not consist entirely of pairs yield smaller bounds. An example is given by the partition leading to the sum

$$
\sum_{k_{i}} \sum_{u_{i}} r_{u_{2}-u_{1}, u_{3}-u_{1}, u_{4}-u_{1}}^{(4)} r_{k_{2}-k_{1}+u_{2}-u_{1}} r_{k_{4}-k_{3}+u_{4}-u_{3}} \prod_{i=1}^{4}\left(w_{k_{i}}^{(n)} \cos k_{i} \lambda\right) \text {. }
$$

Introduce $u_{2}-u_{1}=a, u_{3}-u_{1}=b, u_{4}-u_{1}=c, \alpha=k_{2}-k_{1}, \beta=k_{4}-k_{3}$. Expression (3.19) is then bounded by

$$
\sum_{u_{1}, a, b, c, \alpha, \beta, k_{1}, k_{2}}\left|r_{a, b, c}^{(4)}\right|\left|r_{\alpha+a}\right|\left|r_{\beta+b}\right|\left|w_{k_{1}}^{(n)}\right|\left|w_{k_{1}+\alpha}^{(n)}\right|\left|w_{k_{2}}^{(n)}\right|\left|w_{k_{2}+\beta}^{(n)}\right| .
$$


Summing first over $k_{1}, k_{2}$ leads to the bound

$$
b_{n}^{-2} \sum_{u_{1}, a, b, c, \alpha, \beta}\left|r_{a, b, c}^{(4)}\right|\left|r_{\alpha+a}\right|\left|r_{\beta+b}\right| .
$$

Then sum over $\alpha, \beta$ and then over $a, b, c$ to obtain

$$
b_{n}^{-2} \sum_{u_{1}} 1=m b_{n}^{-2} .
$$

These estimates imply that (3.17) is valid so that Corollary 2 can be applied to obtain the asymptotic normality of such spectral density estimates.

A little reflection shows one that Corollary 3 is still valid if one replaces strict stationarity by stationarity up to eighth order.

If one has a vector-valued strongly mixing strictly stationary process, comparable conditions lead to corresponding results on the asymptotic behavior of cross-spectral estimates (see Brillinger, 1975, and Rosenblatt, 1959). These ideas can be adapted to get results for higher order spectral estimates.

4. Additional remarks. Kolmogorov and Rozanov (1960) have shown a sufficient condition for a Gaussian stationary process to be strongly mixing is that it have a continuous positive spectral density function. In their book, Ibragimov and Rozanov (1978) give an example of a strongly mixing Gaussian process with an unbounded spectral density function. This example is constructed by making use of a result of Helson and Sarason (1967). Non-Gaussian strongly mixing processes can be constructed by taking instantaneous or bandlimited functions of Gaussian strongly mixing processes.

Let $\varphi(z)$ be the standard Gaussian density function and

$$
H_{v}(z)=(-1)^{v} \frac{\left(d^{v} / d z^{v}\right) \varphi(z)}{\varphi(z)},
$$

$v=0, \cdots$, the Hermite polynomials. Let $Y_{1}, \cdots, Y_{m}$ be jointly Gaussian random variables with means zero, variances one, and covariance matrix

$$
\rho=\left(\rho_{i j} ; i, j=1, \cdots, m\right) .
$$

Slepian (1972) has shown that the moment

$$
\begin{aligned}
E\left\{H_{s_{1}}\left(Y_{1}\right) \cdots H_{s_{m}}\left(Y_{m}\right)\right\} & =s_{1} ! \cdots s_{m} ! \Sigma^{\prime} \frac{\rho^{v}}{v !} \\
& =s_{1} ! \cdots s_{m} ! \Sigma^{\prime} \prod_{i<j} \frac{\rho_{i j}^{v_{i j}}}{v_{i j} !}
\end{aligned}
$$

where the primed summation is over all $m \times m$ symmetric matrices $v=\left(v_{i j} ; i, j\right.$ $=1, \cdots, m)$ with nonnegative integer entries such that

$$
s_{k}=\sum_{j \neq k} v_{j k}, k=1, \cdots, m .
$$

This result leads directly to the following result.

CoRollary 4. Let $X=\left(X_{k}\right)$ be a strictly stationary Gaussian sequence with mean zero and summable covariance function. Assume that $\psi(x)$ is a function with 
a Fourier-Hermite expansion that has nonnegative coefficients. Further let

$$
E|\psi(x)|^{2 k}<\infty
$$

for some positive integer $k$. Then the cumulant sequences up to order $2 k$ are summable.

It is easy to generate functions $\psi$ with nonnegative Fourier-Hermite coefficients such that all moments

$$
E|\psi(x)|^{2 m}
$$

are not finite. Consider the identity

$$
e^{t x} e^{-t^{2} / 2}=\sum_{k=0}^{\infty} H_{k}(x) t^{k} / k !
$$

For any positive $c$

$$
\begin{aligned}
\psi(x) & =\left(\frac{2 \pi}{1+c}\right)^{1 / 2} \exp \left\{\frac{x^{2}}{2(1+c)}\right\}=\int_{-\infty}^{\infty} e^{t x} e^{-(1+c) t^{2} / 2} d t \\
& =\sum_{k=0}^{\infty} \frac{H_{k}(x)}{k !} \int_{-\infty}^{\infty} t^{k} e^{-c t^{2} / 2} d t=\sum_{k=0}^{\infty} \frac{H_{2 k}(x)}{2^{k} k !} c^{-k-1 / 2} .
\end{aligned}
$$

With $c$ sufficiently large the $(2 k)$ th moment of $\psi(x)$ will be finite but not higher order moments. If $X=\left(X_{n}\right)$ is a stationary Gaussian sequence with summable covariance sequence and positive spectral density, it will be strongly mixing. The non-Gaussian process $\psi\left(X_{n}\right)$ will be strongly mixing and have cumulant sequences summable up to order $2 k$. If $k=4$ the assumptions of Corollary 3 will be satisfied.

We now show how one can construct examples satisfying the assumptions of Corollary 3 but have a slow rate of decay of the mixing coefficient. A result of Ibragimov and Rozanov (1978, page 181) states that the mixing coefficient of a stationary Gaussian sequence

$$
\alpha(n)=O\left(n^{-\beta}\right)
$$

for a fixed $\beta, 0<\beta<1$, if and only if the process has a spectral density of the form

$$
f(\lambda)=\left|P\left(e^{i \lambda}\right)\right|^{2} w(\lambda)
$$

where $P$ is a polynomial with zeros on $|z|=1$ and $w$ is strictly positive and satisfies a Hölder condition of order $\beta$. One can show that if $\left\{Y_{n}\right\}, E Y_{n} \equiv 0$, is a stationary Gaussian sequence with covariances

$$
r_{n}=|n|^{-1-\beta}
$$

for $|n| \geq 1$, its spectral density is of the form

$$
f(\lambda)=c|\lambda|^{\beta}+g(\lambda)
$$

where $c>0$ is a constant and $g(\lambda)$ an infinitely differentiable function for $|\lambda|$ $<\pi$ (see Zygmund, 1968, vol. 2, page 135). Notice that for $\beta$ in this range

$$
\sum\left|r_{n}\right|<\infty
$$


but not

$$
\sum|n|\left|r_{n}\right|<\infty \text {. }
$$

By choosing $\beta$ sufficiently close to zero, we can effect as slow an inverse polynomial rate of decay of $\alpha(n)$ as may be desired. Consider the process

$$
X_{n}=Y_{n}^{2 k-1}, \quad n=1,2, \cdots,
$$

for $k$ some integer greater than two. By (4.1) it is clear that all cumulant functions are absolutely summable. Corollary 3 is therefore applicable to the process $\left\{X_{n}\right\}$. However, $\left\{X_{n}\right\}$ has exactly the same mixing coefficient sequence as the process $\left\{Y_{n}\right\}$.

One can ask whether absolute summability of cumulants up to fourth order is enough to get the conclusion of Corollary 3. The examples constructed by Herrndorf (1983), indicating that moment conditions up to second order are not enough for a standard version of a central limit theorem, suggest that one may need more than summability of cumulants up to fourth order in the context of spectral density estimates.

\section{REFERENCES}

ANDERSON, T. W. (1971). Statistical Analysis of Time Series. Wiley, New York.

BRILlinger, D. R. and RosenblatT, M. (1967). Asymptotic theory of $k$ th order spectra. Spectral Analysis of Time Series (ed. B. Harris), 153-188. Wiley, New York.

Brillinger, D. R. (1975). Time Series, Data Analysis and Theory. Holt, New York.

Hall, P. and Heyde, C. C. (1980). Martingale Limit Theory and Its Application. Academic, New York.

Hannan, E. J. (1970). Multiple Time Series. Wiley, New York.

Helson, H. and SaRASON, D. (1967). Past and future. Math. Scand. 21 5-16.

HERRNDORF, N. (1983). Stationary strongly mixing sequences not satisfying the central limit theorem. Ann. Probab. 11 809-813.

IbraGimov, I. A. (1962). Some limit theorems for stationary processes. Theory Probab. Appl. 7 349382.

Ibragimov, I. A. and Rozanov, Y. A. (1978). Gaussian Random Processes. Springer, Berlin.

Kolmogorov, A. and ROzANOV, Y. A. (1960). On a strong mixing condition for stationary Gaussian processes. Theory Probab. Appl. 5 204-208.

LEonov, V. P. and SHIRYAEv, A. N. (1959). On a method of calculation of semi-invariants. Theory Probab. Appl. 4 319-329.

Rosenblatt, M. (1956). A central limit theorem and a strong mixing condition. Proc. Nat. Acad. Sci. U.S.A. 42 43-47.

ROSENBLATT, M. (1959). Statistical analysis of stochastic processes with stationary residuals. In Probability and Statistics (ed. U. Grenander) 246-275. Wiley, New York.

RosenblatT, M. (1961). Some comments on narrow band-pass filters. Quart. Appl. Math. 18 387393.

Rosenblatt, M. (1974). Random Processes. Springer, Berlin.

SLEPIAN, D. (1972). On the symmetrized Kronecker power of a matrix and extensions of Mehler's formula for Hermite polynomials. SIAM J. Math. Anal. 3 606-616.

Mathematics Department

University of California, San Diego

La Jolla, California 92093 